\title{
Mathematical modeling of isoflurane action on lamprey spinal
} neurons

\author{
Tamara J Schlichter*1, Anne C Smith ${ }^{2}$, Steven L Jinks ${ }^{2}$ and Timothy J Lewis ${ }^{1}$
}

Address: ${ }^{1}$ Department of Mathematics, University of California, Davis, CA, 95616, USA and 2Department of Anesthesiology and Pain Medicine, University of California, Davis, CA, 95616, USA

Email: Tamara J Schlichter* - tamijoy@math.ucdavis.edu

* Corresponding author

from Seventeenth Annual Computational Neuroscience Meeting: CNS*2008

Portland, OR, USA. 19-24 July 2008

Published: II July 2008

BMC Neuroscience 2008, 9(Suppl I):P26 doi: I0.I |86/I47|-2202-9-SI-P26

This abstract is available from: http://www.biomedcentral.com/I47I-2202/9/SI/P26

(c) 2008 Schlichter et al; licensee BioMed Central Ltd.

Anesthetics are believed to modulate activity of a variety of voltage-gated and ligand-gated ion channels. However, the exact biophysical mechanisms underlying anesthesia remain unclear [1]. Recent experimental evidence suggests that the volatile anesthetic isoflurane targets the TREK and TASK two-pore potassium conductances [2] and the persistent sodium conductance (Jinks et al. unpublished results). Furthermore, recent data indicate that volatile anesthetics produce immobility, a fundamental element of anesthesia, predominantly through direct action at the level of the spinal cord [3].

We use mathematical modeling and experiments on the lamprey spinal cord to study the effects of isoflurane on neuronal activity. Our experimental results on the disinhibited spinal cord preparation suggest that the excitatory interneurons of the CPG are the main target of isoflurane. As the anesthetic concentration increases, ventral root activity (assumed to be representative of the activity of spinal cord excitatory interneurons) transitions from bursting to silent. However, in some animals the activity transitions directly from bursting to silent where in others it transitions from bursting to a brief period of tonic firing before falling silent.

We incorporate the anesthetic-sensitive TREK, TASK and persistent sodium conductances into a preexisting detailed biophysical model of the lamprey excitatory interneuron [4], and a canonical bursting model [5]. We then perform a thorough bifurcation analysis on these models. Our results suggest that the anesthetic effects of
TREK, TASK and persistent sodium currents alone are sufficient to account for the transition from bursting to silent, but are not sufficient to account for a robust transition from bursting to tonic to silent.

\section{Acknowledgements}

Graduate student was partially supported by NSF VIGRE Grant No. DMS0135345

\section{References}

I. Gottschalk A, Haney P: Computational aspects of anesthetic action in simple neural models. Anesthesiology 2003, 98:548-564.

2. Patel AJ, Honore E, Lesage F, Fink M, Romey G, Lazdunski M: Inhalational anesthetics activate two-pore-domain background $\mathrm{K}^{+}$channels. Nat Neurosci 1999, 2(5):422-426.

3. Antognini JF, Schwartz K: Exaggerated anesthetic requirements in the preferentially anesthetized brain. Anesthesiology 1993, 79: $1244-1249$.

4. Brodin L, Traven HGC, Lansner A, Wallen P, Ekeberg O, Grillner S: Computer simulations of $\mathbf{N}$-methyl-D-aspartate receptorinduced membrane properties in a neuron model. I Neurophysiol 1991, 66(2):473-484.

5. Butera RJ, Rinzel J, Smith JC: Models of respiratory rhythm generation in the pre-Botzinger complex. I. bursting pacemaker neurons. J Neurophysiol 1999, 82:382-397. 